\section{Share and share alike?}

\section{Producing a popular} research tool will make you a lot of friends in science. But meeting requests to supply the material puts a heavy burden on your lab. David Cyranoski examines a system under pressure.

Y oshihide Hayashizaki is exasperated. Last year his team published a paper ${ }^{1}$ describing a collection of mouse complementary DNAs (cDNAs) representing the coding sequences of thousands of genes. Now he receives a request to supply these key research tools, on average, almost every day. A new article expanding the collection to some 61,000 cDNAs, published last week, is likely to increase that demand. But in trying to meet these requests, he has stumbled into a logistical, financial and legal minefield. "It's very stressful," says Hayashizaki, who is based at the Genomic Sciences Center in Yokohama, part of RIKEN, Japan's Institute of Physical and Chemical Research.

Hayashizaki's case is extreme. But researchers around the world are facing increasingly burdensome requests for a range of research materials including cDNA clones, antibodies and knockout mice. And they are expected to fulfil them. Many journals, including Nature, require that materials used in the papers that they publish are made available to other researchers. This allows experiments to be repeated, and lets scientists build on each other's achievements rather than starting from square one.

Until recently, mutual goodwill ensured that this almost always happened. But supplying materials can be expensive. The 21,000 cDNAs described in Hayashizaki's first paper cost more than US $\$ 10,000$ to prepare and ship. Journals do not require researchers to make materials available free of charge, but it is unclear how deeply suppliers are expected to dig into their own pockets to cover administrative and other costs. Those involved must also grapple with legal issues surrounding the transfer of materials. And as projects get bigger, materials more expensive and
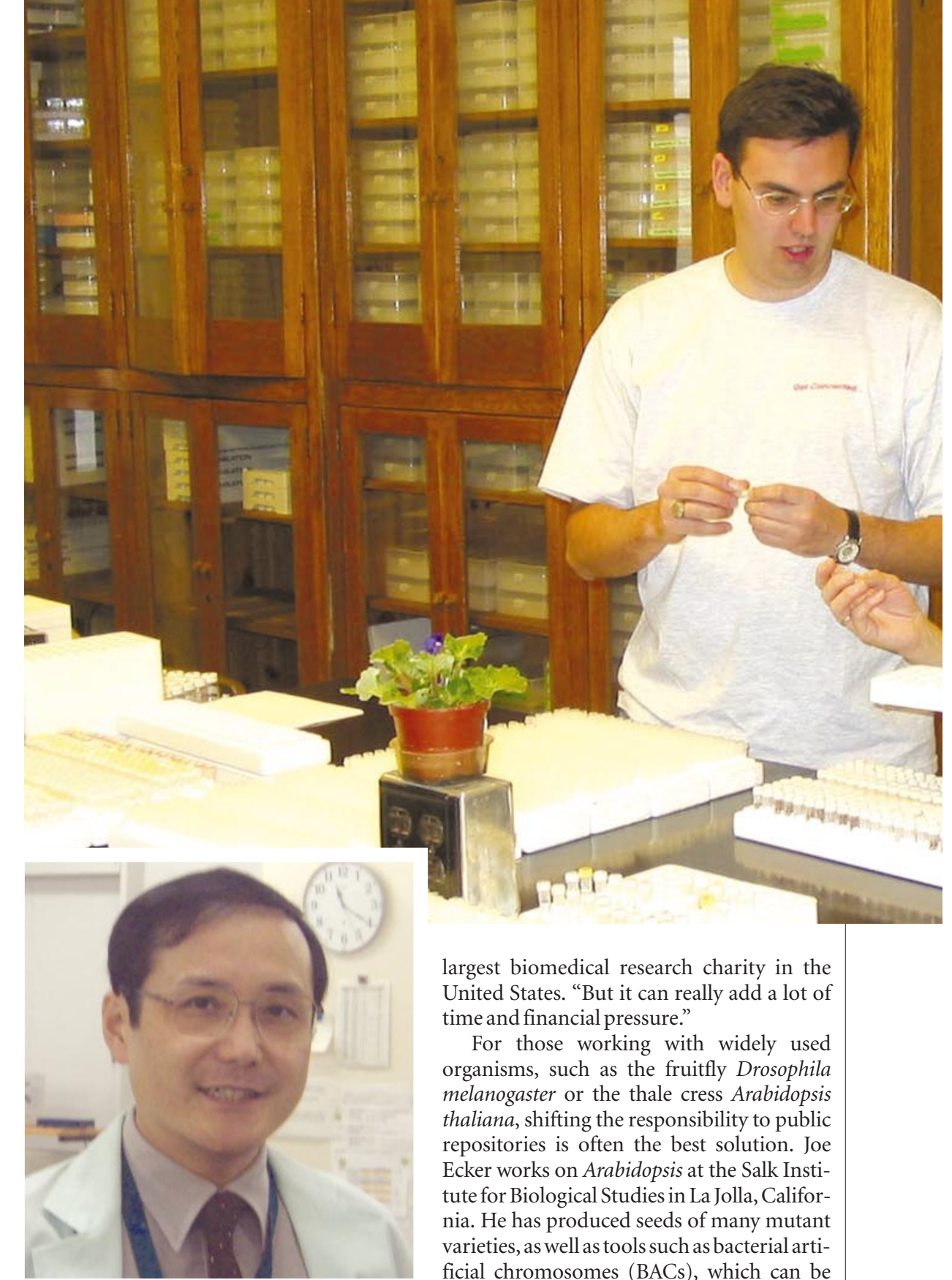

Stressed: for Yoshihide Hayashizaki, sharing his mouse cDNAs has proved a logistical nightmare.

commercial interests more dominant, many researchers are asking how long the current system can continue to function.

Distributing materials is time consuming as well as expensive. Collections must be maintained and orders processed. Shipping itself varies from the trivial task of popping plant seeds into an envelope, to the more intensive chore of putting antibodies on dry ice or express-mailing live animals. Ensuring that the proper materials are sent, and then recouping the costs, can be a full-time job for some technicians or research assistants. "It is an enviable problem, as it means you are getting many citations and getting your name established," says Thomas Cech, president of the Howard Hughes Medical Institute (HHMI) in Chevy Chase, Maryland, the largest biomedical research charity in the time and financial pressure."

For those working with widely used organisms, such as the fruitfly Drosophila melanogaster or the thale cress Arabidopsis thaliana, shifting the responsibility to public repositories is often the best solution. Joe Ecker works on Arabidopsis at the Salk Institute for Biological Studies in La Jolla, California. He has produced seeds of many mutant varieties, as well as tools such as bacterial artificial chromosomes (BACs), which can be used to clone sections of DNA. "The requests would have been a major distraction," says Ecker. "I did not want to turn my group into a stock distribution centre." So Ecker sent his materials to the Arabidopsis Biological Resource Center (ABRC), a collection and distribution service based at Ohio State University in Columbus. The stocks he sent more than doubled the ABRC's holdings, but the extra user fees they generated have so far covered the costs involved.

All would be fine if there were repositories for all areas of science, with each having the money needed to fulfil requests. But BACs and other genomics tools are being created in increasing numbers, and money and space at the repositories is already tight. Mutant-mouse storage houses, for example, are filling up as fast as they are built ${ }^{3}$.

Contracting the job out to the private sector is an alternative for some. Siamon United States. "But it can really add a lot of 


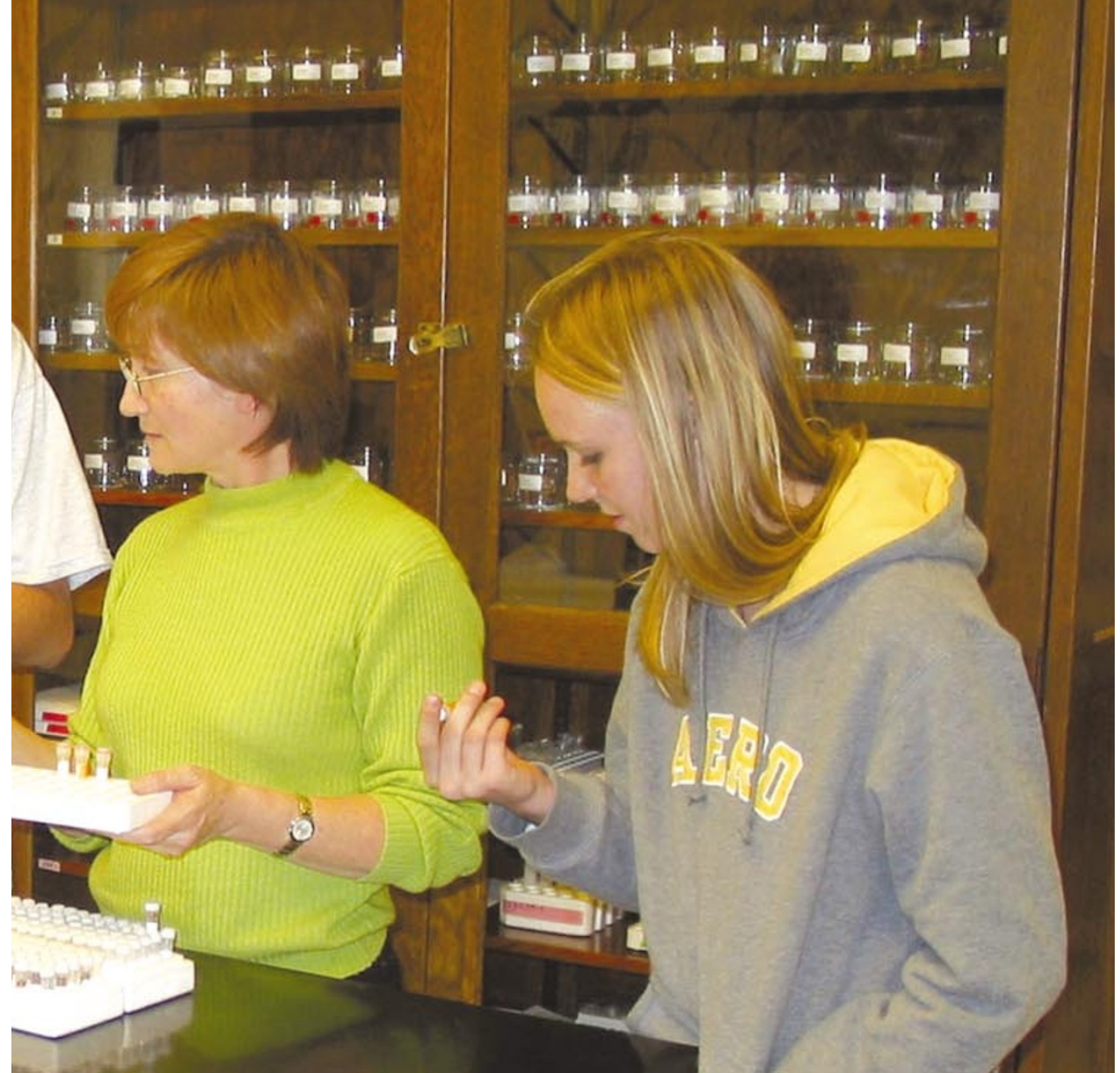

Gordon of the University of Oxford, UK, found that the best way to handle his popular monoclonal antibodies, which have potential therapeutic applications as well as uses in academic research, was to license them to the nearby reagent supplier Serotec, which distributes them for profit. "The arrangement has worked very well," he says. Gordon will also provide materials at his own expense for researchers who cannot afford them. "This does not get specific grant support, but hopefully gets us brownie points," he says.

\section{Supply and demand}

But this option has it drawbacks. Reliance on commercial distributors jacks up the cost of research, limiting the potential pool of users. And it isn't always a viable option, as only the most popular and profitable materials will attract the interest of a distribution company. Commercial suppliers have their place, say scientists, but the funding of public repositories is vital if research tools are to be made as widely available as possible. "We need continued support to allow the distribution of stocks that are important but not necessarily profitable," says Randy Scholl, director of the ABRC.

Even when public or private systems do cover distribution needs, researchers can find themselves thumbing through legal documents that need a lawyer to evaluate. Many researchers, for instance, have complained about the lengthy forms Hayashizaki's insti- tute requires recipients to sign before it will release the clones. Such material transfer agreements (MTAs) are used for a variety of reasons. They can, for example, restrict the ways in which the recipient can use the materials, especially by forbidding potentially dangerous uses such as clinical experiments.

For the sender, MTAs have obvious benefits. They can ensure that the materials do not get degraded through copying, for instance. Ecker says that he once used an MTA to distribute yeast artificial chromosomes, another tool used for cloning DNA. "We simply were saying, 'If you want the clones, get them from us", he says. "Not all MTAs for genomic resources are a bad thing."

But MTAs sometimes contain restrictions relating to publication and property rights that are unacceptable to would-be recipients or their employers. Some agreements, for example, require that the sender receives a part of the profits from any commercialization of the research, or that any potential publication must be authorized by the sender. "For almost every MTA, there are some researchers whose institution will not allow them to sign it and thus receive the associated material," says Scholl.

Thankfully, researchers are trying to minimize the use of such agreements. "We try to ignore MTAs when sending," says Claude Desplan, a developmental biologist at New York University, who supplies mutant lines of Drosophila. When sending his seed stocks and

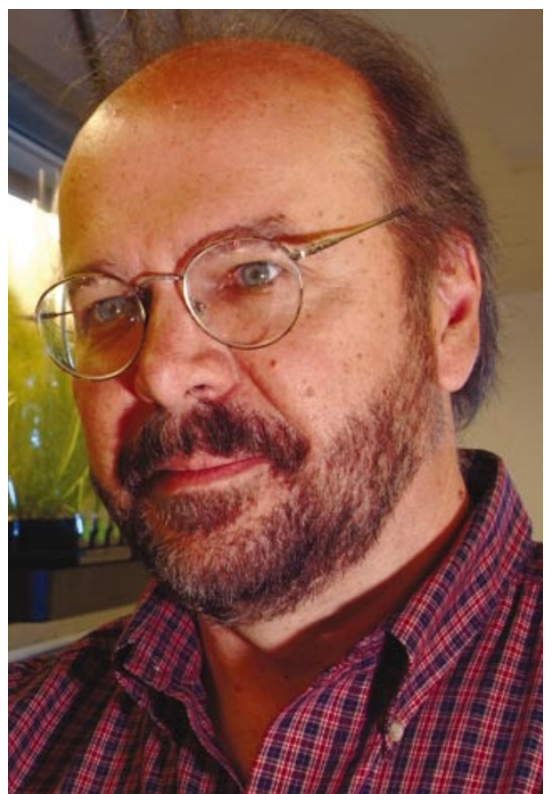

Taking stock: Joe Ecker (above) solved his distribution problems through the Arabidopsis Biological Resource Center (left).

BACs to the ABRC, Ecker says he convinced administrators at the Salk Institute that his project would have "the greatest impact on the plant-biology community with no strings attached", and so was able to avoid using an MTA. Cech adds that the HHMI's form is only about three sentences long.

Repositories, especially in the United States, are also taking action, putting pressure on collections elsewhere to follow suit. The Mammalian Gene Collection, a set of cDNAs supplied by the National Institutes of Health (NIH) in Bethesda, Maryland, does not use MTAs, and the ABRC this year decided to refuse any further donations with MTAs attached. "This will place the burden of distribution of these large and unwieldy collections squarely on the shoulders of the individual investigators who have produced them," says Ecker. "This could force institutions to reduce restrictions on genomic-scale materials.”

\section{Ripped red tape}

Indeed, some repositories have found themselves forced to drop MTAs. In November 2001, the RZPD, Germany's human-genome resource centre in Berlin, which recently began distributing a valuable library of human cDNAs, shifted from a lengthy MTA to a brief "good faith agreement". This merely frees the RZPD from responsibility for any problems with the material itself or dangerous uses of it. The move was prompted by the realization that its old MTA was forcing researchers in Germany to obtain resources from institutions in the United States.

Even if researchers can get around MTAs, other legal problems sometimes await - as Hayashizaki has found out. Mouse cDNAs are created from the messenger RNA (mRNA) 
